1. Vivier E, et al. Innate or adaptive immunity? The example of natural killer cells. Science. 2011; 331(6013):44-49.

2. Raulet DH, Vance RE. Self-tolerance of natural killer cells. Nat Rev Immunol. 2006;6(7):520-531.

3. Yokoyama WM, Kim S. How do natural killer cells find self to achieve tolerance? Immunity. 2006 24(3):249-257.

4. Brodin P, Hoglund P. Beyond licensing and disarm ing: a quantitative view on NK-cell education. EurJ Immunol. 2008;38(11):2934-2937.

5. Orr MT, Lanier LL. Natural killer cell education and tolerance. Cell. 2010;142(6):847-856

6. Bix M, Liao N-S, Zijlstra M, Loring J, Jaenisch R, Raulet D. Rejection of class I MHC-deficient haemopoietic cells by irradiated MHC-matched mice. Nature. 1991;349(6307):329-331.

7. Liao N-S, Bix M, Zilstra M, Jaenish R, Raulet D. MHC class I deficiency: susceptibility to natural killer (NK) cells and impaired NK activity. Science. 1991;253(5016):199-202.

8. Hoglund P, et al. Recognition of beta 2-microglobulin-negative (beta $2 \mathrm{~m}$-) T-cell blasts by natural killer cells from normal but not from beta $2 \mathrm{~m}$ mice: nonresponsiveness controlled by beta $2 \mathrm{~m}$ bone marrow in chimeric mice. Proc Natl Acad Sci US A. 1991;88(22):10332-10336.

9. Kim S, et al. Licensing of natural killer cells by host major histocompatibility complex class I molecules. Nature. 2005;436(7051):709-713.

10. Fernandez NC, Treiner E, Vance RE, Jamieson AM, Lemieux S, Raulet DH. A subset of natural killer cells achieves self-tolerance without expressing inhibitory receptors specific for self-MHC molecules. Blood. 2005;105(11):4416-4423.

11. Anfossi N, et al. Human NK cell education by inhibitory receptors for MHC class I. Immunity. 2006; 25(2):331-342

12. Guia $S$, et al. Confinement of activating receptors at the plasma membrane controls natural killer cell tolerance. Sci Signal. 2011;4(167):ra21.

13. Elliott JM, Wahle JA, Yokoyama WM. MHC class Ideficient natural killer cells acquire a licensed phenotype after transfer into an MHC class I-sufficient environment. J Exp Med. 2010;207(10):2073-2079.

14. Joncker NT, Shifrin N, Delebecque F, Raulet DH.
Mature natural killer cells reset their responsiveness when exposed to an altered MHC environment. J Exp Med. 2010;207(10):2065-2072.

15. Tarek N, et al. Unlicensed NK cells target neuroblastoma following anti-GD2 antibody treatment. J Clin Invest. 2012;122(9):3260-3270.

16. Parham P. MHC class I molecules and KIRs in human history, health and survival. Nat Rev Immunol. 2005;5(3):201-214.

17. Orr MT, Murphy WJ, Lanier LL. 'Unlicensed' natural killer cells dominate the response to cytomegalovirus infection. Nat Immunol. 2010;11(4):321-327.

18. Kohrt HE, et al. CD137 stimulation enhances the antilymphoma activity of anti-CD20 antibodies. Blood. 2011;117(8):2423-2432.

19. Kohrt HE, et al. Stimulation of natural killer cells with a CD137-specific antibody enhances trastuzumab efficacy in xenotransplant models of breast cancer. J Clin Invest. 2012;122(3):1066-1075

20. Romagne F, et al. Pre-clinical characterization of 1-7F9, a novel human anti-KIR therapeutic antibody that augments NK-mediated killing of tumor cells. Blood. 2009;114(13):2667-2677.

\title{
NPARM in PHOX2B: why some things just should not be expanded
}

\author{
Michael D. Gershon
}

Department of Pathology and Cell Biology, Columbia University, New York, New York, USA.

\begin{abstract}
Although the neural crest and its derivatives have been studied for a very long time, disorders of derivatives of the crest, the neurocristopathies, are not well understood. In this issue of the JCI, Nagashimada et al. provide an elegant analysis of one neurocristopathy, the association of neuroblastoma (NB) with Hirschsprung disease (HSCR) (aganglionosis of the terminal bowel) and congenital central hypoventilation syndrome (CCHS) (also known as NB-HSCR-CCHS), linked to mutations in PHOX2B. In a mouse model, Nagashimada et al. demonstrate that a disease-linked mutation promotes tumorigenesis and disrupts neurogenesis, sympathetic gangliogenesis, and crest cell colonization of the terminal bowel. They also show that mutant PHOX2B results in decreased proliferation of crest-derived cells and the development of glia at the expense of neurons. The work raises intriguing issues about the possible common origin of sympathetic and enteric nervous systems and provides new hope that we may someday understand the vexing abnormalities in gastrointestinal function that persist after the surgical treatment of HSCR.
\end{abstract}

The neural crest has long been recognized to be a gift that evolution has given to developmental biologists. It is a transient structure comprising cells that are probably heterogeneous (1), but that, as a unit, is multipotent, giving rise to melanocytes, Schwann cells, sympathetic neurons, parasympathetic neurons, enteric neurons,

Conflict of interest: The author has declared that no conflict of interest exists.

Citation for this article: $J$ Clin Invest. 2012; 122(9):3056-3058. doi:10.1172/JCI63884. enteric glia, endocrine cells, fibroblasts, muscle, bone, cartilage, and meninges (2). The neural crest thus provides opportunities to study the cellular and molecular mechanisms of epithelial-mesenchymal transformation (and the reverse), migration, aggregation, and differentiation. It has been a gift that just keeps on giving. Despite the developmental biological treasure that the neural crest has provided, however, the disorders that stem from neural crest dysfunction, the neurocristopathies (3), remain vexatious.
In this issue, Nagashimada et al. (4), in a truly remarkable study, have now provided a real insight into how a mutation in $P H O X 2 B$, a gene that encodes a paired homeodomain transcription factor that plays a critical role in the development of crest-derived autonomic neurons (5), can act in different cells in a gain-of-function or in a loss-of function manner. The gainof-function activity is tumorigenic, causing neuroblastomas (NBs) to arise in the sympathetic nervous system, while perversely synergizing with the loss-of-function effect to disrupt neurogenesis, sympathetic gangliogenesis, and crest cell colonization of the terminal bowel, which becomes aganglionic (Hirschsprung disease [HSCR]). The faults in formation of neurons and ganglia appear to be the result of a failure of the reciprocal inactivation of $\mathrm{PHOX} 2 \mathrm{~B}$ and SOX10.

During normal development, crestderived precursors of sympathetic and enteric neurons initially express SOX10, but acquire PHOX2B when they enter the preaortic (6) or enteric mesenchyme (7-9). Bipotent progenitors express both PHOX2B and SOX10, but SOX10 is inactivated in cells that are destined to form neurons, and PHOX2B is inactivated in 


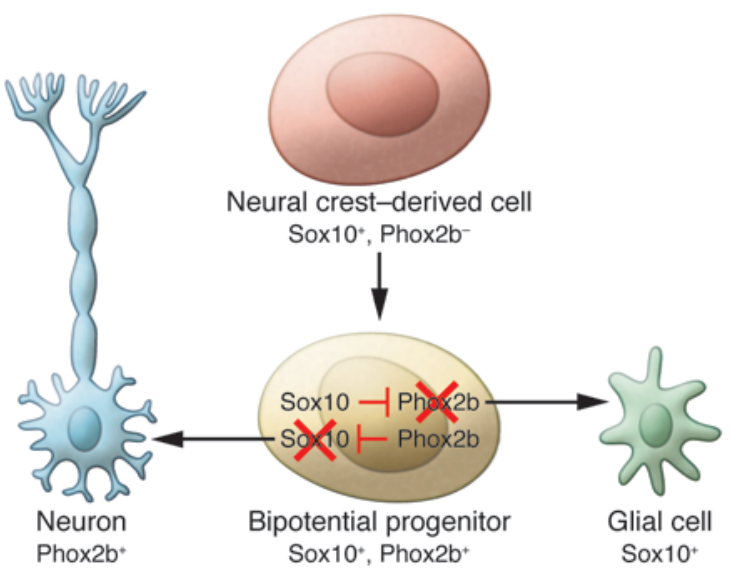

Figure 1

The role of $\mathrm{PHOX} 2 \mathrm{~B}$ in maintaining a balance in glial and neuronal differentiation. During normal development, crest-derived precursors of sympathetic and enteric neurons initially express SOX10 but acquire PHOX2B when they enter the preaortic or enteric mesenchyme. These bipotent progenitors express both $P H O X 2 B$ and SOX10, but SOX10 is inactivated in cells that are destined to form neurons, while $P H O X 2 B$ is inactivated in cells that give rise to glia. Nagashimada et al. demonstrate that the balance between PHOX2B and SOX10 is the result of their mutual and reciprocal suppression. Mutation in $P H O X 2 B$ upsets this balance and weakens the inactivation of SOX10, which inhibits early proliferation and biases differentiation of progenitors toward the glial fate at the expense of the neuronal lineage.

cells that give rise to glia (10). Nagashimada et al. (4) demonstrate that the balance between PHOX2B and SOX10 is evidently the result of their mutual and reciprocal suppression. Mutation in $\mathrm{PHOX} 2 \mathrm{~B}$ upsets this balance and weakens the inactivation of SOX10, which inhibits early proliferation and biases differentiation of progenitors toward the glial fate at the expense of the neuronal lineage (Figure 1). As if disrupted sympathetic ganglia, HSCR, and NBs were not enough, the mutation also interferes with the development of parafacial brainstem neurons, which are involved in the control of respiration, leading to congenital central hypoventilation syndrome (CCHS), or Ondine's curse, in which respiratory arrest occurs during sleep (11, 12). Affected individuals thus suffer from an unholy trinity of NB-HSCR-CCHS, which dooms them to a precarious life of frequent surgeries, tethering to medical machines, and imperfect treatment.

\section{Identifying the genetic cause of disease}

Most of the PHOX2B mutations found in patients with the NB-HSCR-CCHS association are missense or open reading frameshifting nucleotide deletions/insertions $(13,14)$. The PHOX2B protein contains a single homeodomain and two polyalanine repeat sequences. An expansion of the sec- ond polyalanine repeat is associated with CCHS, but mutations leading to nonpolyalanine repeat expansion mutations (NPARMs) in PHOX2B accompany the NB-HSCR-CCHS association $(5,15)$. The observation that NPARMs in PHOX2B accompany the NB-HSCR-CCHS association was exciting; however, a correlation cannot, by itself, establish causation. A remarkable feature of the report of Nagashimada et al. (4) is that it comes very close to making that leap.

Nagashimada et al. (4) did not say so, but their work can be thought of as a molecular genetic analog of Koch's postulates, which provide criteria for establishing causation of infectious diseases (16). The NB-HSCR-CCHS association, of course, is not an infectious disease, but the analogy is apt. Koch's first and second postulates stipulate that the putative organism is found in, and isolated from, virtually every individual in whom the disease is manifest, but is not found in individuals in whom it is not manifest (although Koch modified his postulates to include carriers who harbor the organism but do not become ill). In the molecular genetic analogy, NPARMs in PHOX2B, rather than an organism, are found in patients with the NB-HSCR-CCHS association, and the NB-HSCR-CCHS association is not seen in individuals that lack NPARMs. Koch's third and fourth postulates stipulate that the putative organism mimics the disease it is suspected of causing when injected into a healthy organism and then that it may be isolated from the experimentally infected subject. In molecular genetic terms, Nagashimada et al. (4) have now taken the mutant NPARM form of human $P H O X 2 B$ and introduced it into the mouse Phox $2 b$ locus. When that is done, clinical features of the NB-HSCR-CCHS association are recapitulated in the transgenic mouse. Unfortunately, the recapitulation is not perfect in that the transgenic mice display disrupted sympathetic ganglia, HSCR, and CCHS, but not NBs. Mice, however, are not human, and the heterozygous mice bearing the NPARM in the Phox2b locus may not survive long enough to acquire NBs. Nagashimada et al. present their data with suitable qualification, but their work is strong evidence that NPARM in PHOX2B causes the NB-HSCR-CCHS association.

\section{Developmental insight}

Nagashimada et al. (4) also contribute to our understanding of the role of $\mathrm{PHOX} 2 \mathrm{~B}$ in development. They show that NPARM PHOX2B is a dominant negative inhibitor of the transactivation of the dopamine $\beta$-hydroxylase $(D B H)$ promoter by wildtype PHOX2B and that NPARM causes PHOX2B to repress rather than transactivate a Sox 10 enhancer. Those observations provide critical documentation of the reciprocal actions of $\mathrm{PHOX} 2 \mathrm{~B}$ and SOX 10 during autonomic development. When the ability of PHOX2B to suppress SOX10 is compromised, not only is gliogenesis enhanced and neurogenesis reduced, but also the early proliferation of precursors in the anlagen of enteric and sympathetic ganglia is reduced. Continued proliferation of precursors is particularly important in the gut, where colonization of the entire bowel depends on it. Premature differentiation of enteric crest-derived cells has previously been implicated in their failure to colonize the entire bowel when endothelin 3/endothelin B signaling is compromised $(10,17$, 18). Precursors proliferate, but neurons do not. A sufficiently large population of crest-derived precursors of vagal (the largest source) and sacral origin is necessary to colonize the entire bowel (19). Because proliferation within the enteric mesenchyme is essential to achieving the critical number of crest-derived cells to complete the job of getting to the bottom of the bowel, it is easy to understand why an NPARM in 
PHOX2B that interferes with the proliferation of enteric crest-derived cells at an early stage of development might lead to the terminal aganglionosis of HSCR.

\section{The making of a nervous system}

The experiments of Nagashimada et al. (4) also provide interesting support for an old suggestion that sympathetic and enteric neurons may be derived from a common intermediate progenitor (20). This idea arose, in part, from observations that transiently catecholaminergic (TC) cells appear early during the development of the gut in rats (E11-E14) and mice (E10-E13) (21-24). These cells, which express neural crest markers, such as $\mathrm{p} 75^{\mathrm{NTR}}$, and neuronal markers, including neurofilament proteins, actively proliferate (22). TC cells, therefore, are clearly not neurons, although they are probably committed to the neuronal lineage. In fact, the many catecholaminergic markers that TC cells express, including tyrosine hydroxylase, $\mathrm{DBH}(22)$, the norepinephrine transporter (NET) $(25,26)$, and fetal sympathoadrenal differentiation antigens $(20,27)$, are also expressed by the permanently catecholaminergic sympathetic neurons, TC cells, and sympathetic neurons; moreover, both depend on expression of the Ascl1 transcription factor (28-30). As development progresses, TC cells disappear because they give rise to noncatecholaminergic neurons, including all of the serotonergic neurons of the enteric nervous system (ENS) (30). Although the terminally differentiated neuronal successors of TC cells lack catecholamines, they continue to express a limited set of the catecholaminergic properties of their fetal predecessors, including DBH (22) and NET (26). The significance of this continued expression is not entirely clear, but it has been noted that TC cellderived enteric serotonergic neurons, which express both DBH and NET, are deficient in mice that lack NET. It thus may be more than coincidental that $\mathrm{DBH}$ is a target of PHOX2B and that Nagashimada et al. (4) report that NPARM in PHOX2B exerts a dominant negative effect that impairs transactivation of the $D B H$ promoter by wild-type PHOX2B.

\section{Remaining questions}

The roles that DBH and NET play in ENS development and the significance for the ENS of PHOX2B transactivation of $\mathrm{DBH}$ require further investigation. It would probably be useful to look at the phenotypes of the neurons in the proximal ganglionated bowel in patients with HSCR, particularly those with the NBHSCR-CCHS association. Excision of the aganglionic bowel in patients with HSCR is lifesaving, but the function of the gut after surgery is often disappointing. These results may well be the result of abnormalities of neuronal development in the ganglionated bowel. The ENS is complex, and the mere presence of ganglia is not necessarily evidence of normal development and function. The work of Nagashimada et al. (4) thus provides hints for new research as well as insights into the derivation of the NB-HSCR-CCHS association.

\section{Acknowledgments}

This work was supported by grants from the NIH (NS12969 and NS15547).

Address correspondence to: Michael D. Gershon, Department of Pathology and Cell Biology, Columbia University, P\&S, 630 West 168th Street, New York, New York 10032, USA. Phone: 212.305.3447; Fax: 212.305.3790; E-mail: mdg4@columbia.edu.

1. Henion PD, Weston JA. Timing and pattern of cell fate restrictions in the neural crest lineage. Development. 1997;124(21):4351-4359.

2. Le Douarin NM, Kalcheim C. The Neural Crest. 2nd ed. Cambridge, United Kingdom: Cambridge University Press; 1999.

3. Bolande RP. Neurocristopathy: its growth and development in 20 years. Pediatr Pathol Lab Med. 1997;17(1):1-25.

4. Nagashimada $M$, et al. Autonomic neurocristopathy-associated mutations in PHOX2B dysregulate Sox10 expression. J Clin Invest. 2012; 122(9):3145-3158

5. Pattyn A, Morin X, Cremer H, Goridis C, Brunet $\mathrm{J}-\mathrm{F}$. The homeobox gene Phox $2 \mathrm{~b}$ is essential for the development of autonomic neural crest derivatives. Nature. 1999;399(6734):366-370.

6. Ernsberger U, Reissmann E, Mason I, Rohrer H The expression of dopamine beta-hydroxylase, tyrosine hydroxylase, and Phox 2 transcription factors in sympathetic neurons: evidence for common regulation during noradrenergic induction and diverging regulation later in development. Mech Dev. 2000;92(2):169-177.

7. Anderson RB, Stewart AL, Young HM. Phenotypes of neural-crest-derived cells in vagal and sacral pathways. Cell Tissue Res. 2006;323(1):11-25.

8. Young HM, et al. Dynamics of neural crest-derived cell migration in the embryonic mouse gut. Dev Biol. 2004;270(2):455-473.

9. Corpening JC, Cantrell VA, Deal KK, SouthardSmith EM. A Histone2BCerulean BAC transgene identifies differential expression of Phox $2 b$ in migrating enteric neural crest derivatives and enteric glia. Dev Dyn. 2008;237(4):1119-1132.

10. Sasselli V, Pachnis V, Burns AJ. The enteric nervous system. Dev Biol. 2012;366(1):64-73

11. Dubreuil V, et al. A human mutation in Phox $2 b$ causes lack of $\mathrm{CO}_{2}$ chemosensitivity, fatal central apnea, and specific loss of parafacial neurons. Proc Natl Acad Sci U S A. 2008;105(3):1067-1072.

12. Trochet D, et al. PHOX2B genotype allows for prediction of tumor risk in congenital central hypoventilation syndrome. Am J Hum Genet. 2005; 76(3):421-426.

13. Amiel J, et al. Polyalanine expansion and frameshift mutations of the paired-like homeobox gene PHOX2B in congenital central hypoventilation syndrome. Nat Genet. 2003;33(4):459-461.

14. Trochet D, et al. Molecular consequences of PHOX2B missense, frameshift and alanine expansion mutations leading to autonomic dysfunction. Hum Mol Genet. 2005;14(23):3697-3708.

15. Dubreuil V, et al. Defective respiratory rhythmogenesis and loss of central chemosensitivity in Phox $2 \mathrm{~b}$ mutants targeting retrotrapezoid nucleus neurons. J Neurosci. 2009;29(47):14836-14846.

16. Falkow S. Molecular Koch's postulates applied to microbial pathogenicity. Rev Infect Dis. 1988; 10(suppl 2):S274-S276.

17. Wu JJ, Chen J-X, Rothman TP, Gershon MD. Inhibition of in vitro enteric neuronal development by endothelin-3: mediation by endothelin B receptors. Development. 1999;126(6):1161-1173.

18. Druckenbrod NR, Epstein ML. Age-dependent changes in the gut environment restrict the invasion of the hindgut by enteric neural progenitors. Development. 2009;136(18):3195-3203.

19. Barlow AJ, Wallace AS, Thapar N, Burns AJ. Critical numbers of neural crest cells are required in the pathways from the neural tube to the foregut to ensure complete enteric nervous system formation. Development. 2008;135(9):1681-1691.

20. Carnahan JF, Anderson DJ, Patterson PH. Evidence that enteric neurons may derive from the sympathoadrenal lineage. Dev Biol. 1991;148(2):552-561.

21. Baetge G, Gershon MD. Transient catecholaminergic (TC) cells in the vagus nerves and bowel of fetal mice: relationship to the development of enteric neurons. Dev Biol. 1989;132(1):189-211.

22. Baetge G, Pintar JE, Gershon MD. Transiently catecholaminergic (TC) cells in the bowel of fetal rats and mice: Precursors of non-catecholaminergic enteric neurons. Dev Biol. 1990;141(2):353-380.

23. Teitelman G, Joh TH, Reis DJ. Transient expression of a noradrenergic phenotype in cells of the rat embryoinic gut. Brain Res. 1978;158(1):229-234.

24. Cochard P, Goldstein M, Black IB. Ontogenetic appearance and disappearance of tyrosine hydroxylase and catecholamines. Proc Natl Acad Sci U S A. 1978;75(6):2986-2990.

25. Gershon MD, Rothman TP, Joh TH, Teitelman GN. Transient and differential expression of aspects of the catecholaminergic phenotype during development of the fetal bowel of rats and mice. J Neurosci. 1984;4(9):2269-2280.

26. Li ZS, Caron MG, Blakely RG, Margolis KG, Gershon MD. Dependence of serotonergic and other nonadrenergic enteric neurons on norepinephrine transporter expression. J Neurosci. 2010; 30(49):16730-16740.

27. Carnahan JF, Patterson PH. The generation of monoclonal antibodies that bind preferentially to adrenal chromaffin cells and the cells of embryonic sympathetic ganglia. J Neurosci. 1991;11(11):3493-3506.

28. Lo L-C, Johnson JE, Wuenschell CW, Saito T, Anderson DJ. Mammalian achaete-scute homolog 1 is transiently expressed by spatially restricted subsets of early neuroepithelial and neural crest cells. Genes Dev. 1991;5(9):1524-1537.

29. Lo L, Dormand E, Greenwood A, Anderson DJ. Comparison of the generic neuronal differentiation and neuron subtype specification functions of mammalian achaete-scute and atonal homologs in cultured neural progenitor cells. Development. 2002; 129(7):1553-1567.

30. Blaugrund E, et al. Distinct subpopulations of enteric neuronal progenitors defined by time of development, sympathoadrenal lineage markers, and Mash-1dependence. Development. 1996;122(1):309-320. 